\title{
essai de constitution et d'utilisation d'une banque de données sur le sous-sol d'une plaine alluviale (région de Grenoble, France)
}

\author{
par \\ D. Fabre \\ J. Letellier \\ I.R.I.G.M. Université de Grenoble, 38041 Grenoble cedex \\ G. Mercieca
}

E.N.S.T.P. Yamassoukro, Côte d'Ivoire

\section{Cadre général}

Le développement urbain du grand Grenoble est conditionné par une morphologie particulière héritée des glaciations quaternaires. La ville de Grenoble et ses principales banlieues (St-Martin-d'Hères, Échirolles, Fontaine...) constituent une agglomération de 500000 habitants qui s'étend essentiellement sur la surface horizontale d'un fond de vallée, au confluent de I'Isère et du Drac, "I'Y grenoblois" (fig. 1).

Les alluvions réçentes de ces deux rivières, à dominante fine pour I'Isère, plus grossière pour le Drac, recouvrent des terrains quaternaires plus anciens :

- les argiles d'Eybens, déposées dans un très important lac d'ombilic à I'interglaciaire Riss-Würm et qui n'ont été que partiellement érodées par la crue würmienne;

- les formations de fond, beaucoup plus grossières, constituées par des moraines rissiennes et des alluvions anciennes, La totalité de cet ensemble de terrains meubles représente une épaisseur très importante qui varie probablement entre 300 et 500 mètres (plus de $400 \mathrm{~m}$ au sondage de Beauvert, 310 et $500 \mathrm{~m}$ d'après deux profils sismiques effectués l'un à l'amont, l'autre à l'aval de Grenoble, in Fourneaux, 1976).

A l'aménagement des fonds de vallée est venu par ailleurs s'ajouter depuis une dizaine d'années la colonisation progressive des versants, ce qui a obligé le département de l'Isère à prendre des dispositions rigoureuses pour la délimitation des zones de risques naturels (Tachker, 1973).

Depuis 1970, I'Université de Grenoble a entrepris le recensement des données géotechniques sur le sous-sol régional et des synthèses partielles (Couturier, 1974; Margalhan-Ferrat, 1975; Bozonnet, 1978; Talloni, 1978) ont permis l'établissement de plusieurs cartes géotechniques au 1/25000 couvrant la presque totalité des zones basses. Une synthèse au 1/50000 sur l'ensemble de la région est actuellement en cours (Letellier, à paraître). Ces études sont menées en relation avec l'Agence d'Urbanisme de la Région grenobloise (A. U.R. G.).

La présente note traite du stockage informatique des données et de l'utilisation pratique de celles-ci.

\section{Conception générale de la banque de données}

L'initiative du traitement informatique des données de sondages dans la région grenobloise revient à G. Biguenet, M. Dayre et J. L. Favre (1970). Mais ce. n'est véritablement qu'après 1976 que le recensement et le stockage systématique ont été entrepris (Mercieca, 1977). Plus de 2000 sondages sont aujourd'hui enregistrés.

\subsection{Collecte des données et organisation des fichiers}

Les données sont regroupées à partir de dossiers d'origine très diverse. A ce niveau, l'intervention du géologue est indispensable pour effectuer un examen critique, clarifier les appellations des sondeurs et évaluer la précision sur la localisation géographique et les profondeurs. Un code est employé pour les informations sémantiques. Pour des raisons d'organisation du stockage, les données relatives à un même sondage ont été scindées en plusieurs fichiers selon leur nature. Un sondage mécanique alimente ainsi :

- le fichier situation (fig. 2), réunissant les données géographiques et les références administratives du sondage;

- le fichier lithologie, qui regroupe les informations géologiques en fonction de la profondeur et les caractéristiques techniques du sondage;

- les fichiers identification et propriétés mécaniques, qui sont ouverts lorsque le sondage a permis le 


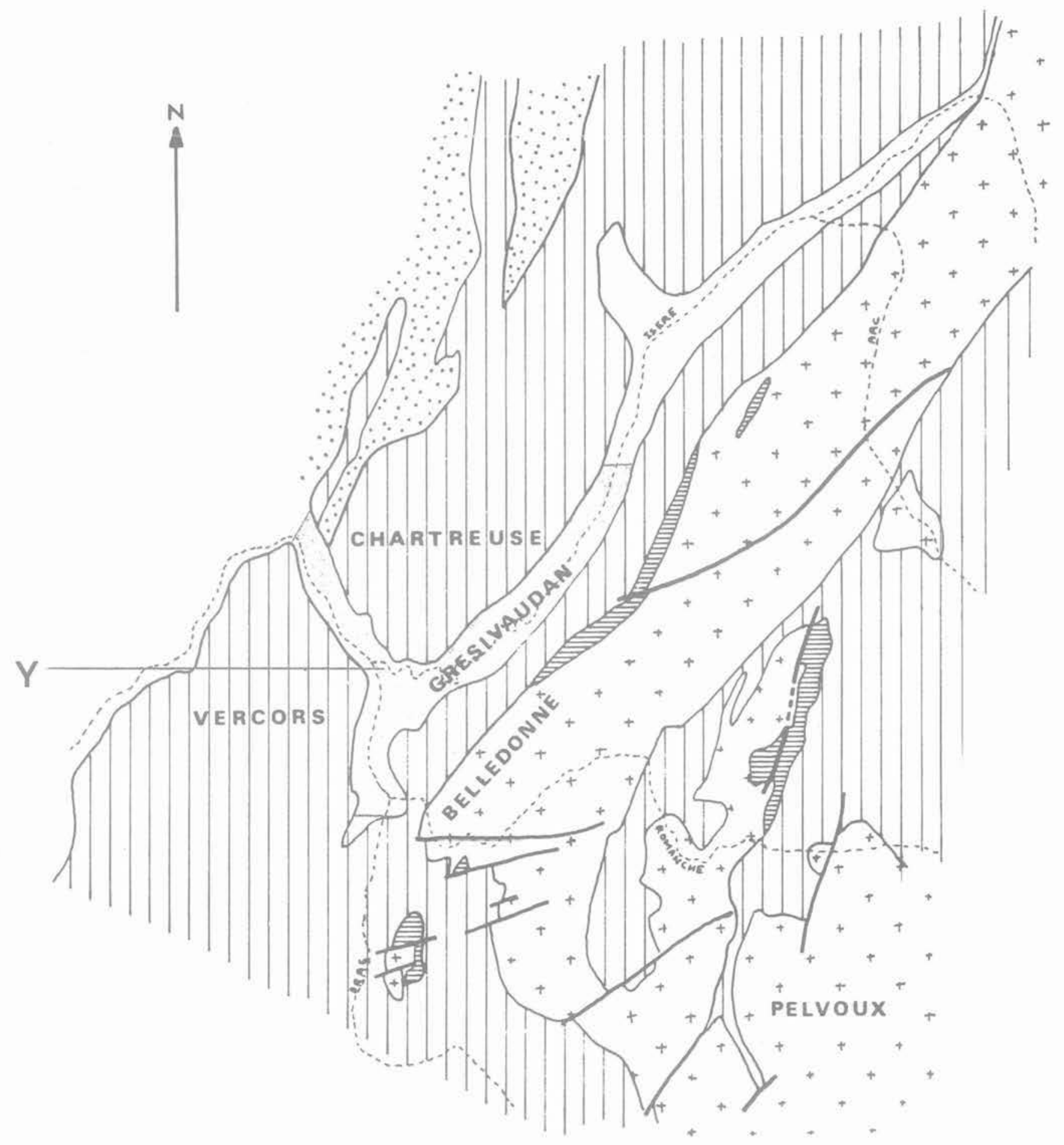

QUATERNAIRE

$\because$ TERTIAIRE

III SECONDAIRE

Echelle

PERMO-CARBONIFERE

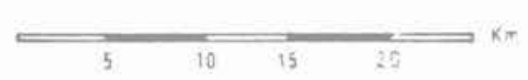

$++_{+-}^{+}$MASSIFS CRISTALLINS EXTERNES

Fig. 1 Le cadre géologique de l'Y grenoblois, d'après B. Couturier (1974) 


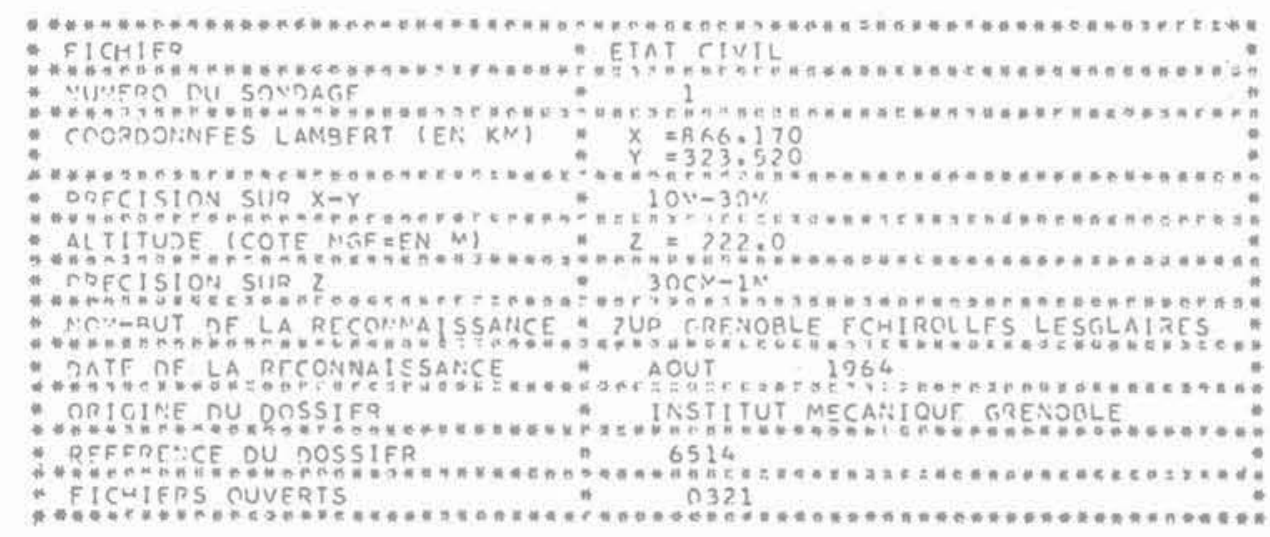

Fig. 3 Exemple d'édition des informations à l'aide du programme IMPR1

prélèvement d'échantillons et des analyses ou essais en laboratoire.

\subsection{Les programmes de traitement}

Les données étant chargées sur un disque magnétique, différents programmes d'accès et d'édition ont été conçus.

\subsubsection{Les programmes d'édition}

Le programme IMPR 1 peut répondre aux besoins de l'ingénieur, de l'architecte ou de l'urbaniste intéressé par la connaissance du sous-sol à proximité de l'emplacement d'un projet. II permet la recherche des sondages dans une zone géographique donnée et édite leurs numéros et leurs caractéristiques administratives (nom de l'entreprise de sondage, date et but de la reconnaissance...) (fig. 3).

Le programme IMPR 2 permet la recherche et l'édition à partir du numéro de sondage des données lithologiques; les programmes IMPR 3 et IMPR 4 travaillent de la même façon sur les données géotechniques.

\subsubsection{Les programmes de sorties graphiques}

Le programme POZON permet le positionnement sur un plan à une échelle choisie de l'implantation des sondages (avec ou sans numéro de stockage). Le quadrillage géographique (coordonnées Lambert) est indiqué à demande.

Le programme ISOPA indique sur un plan l'épaisseur des sols fins de surface ou la cote du premier niveau de formations grossières.
Le programme GEOL permet d'obtenir par un figuré spécial à l'emplacement du sondage la nature du sous-sol sous la couche de terre végétale ou à une profondeur ou une cote N.G.F. donnée (fig. 4).

Enfin, le programme COUPE édite les "logn de sondage sous forme symbolique selon un cheminement passant par des sondages. L'interprétation et le tracé de la coupe complète du sous-sol selon ce cheminement est laissée au géologue. Cette limite imposée à l'outil informatique se justifie d'abord par la nature lenticulaire complexe des dépôts alluviaux. Celle-ci implique des distributions spatiales et des épaisseurs extrêmement variables et interdit pratiquement l'interpolation automatique entre les données. Mais cette façon de procéder nous apparaît également satisfaisante sur le plan philosophique car elle oblige à un contrôle technique minutieux de la qualité des résultats produits et permet d'écarter les interprétations basées sur un trop petit nombre de données ou des données incertaines.

\section{Exemple d'utilisation}

\subsection{Nature de la demande}

L'Agence d'Urbanisme de la Région grenobloise, qui a pour mission d'aider les communes à concevoir leur programme d'urbanisation et à réaliser leurs plans d'occupation des sols (P.O.S.), s'intéresse naturellement à la connaissance du sous-sol des zones non encore urbanisées. Dans le cas des études préliminaires, faites en général sans moyens de reconnaissance spécifiques, le recours à la banque de données paraît très utile. Nous donnerons comme exemple le cas d'un terrain de 40 hectares non encore équipé situé sur une commune limitrophe de Grenoble. 


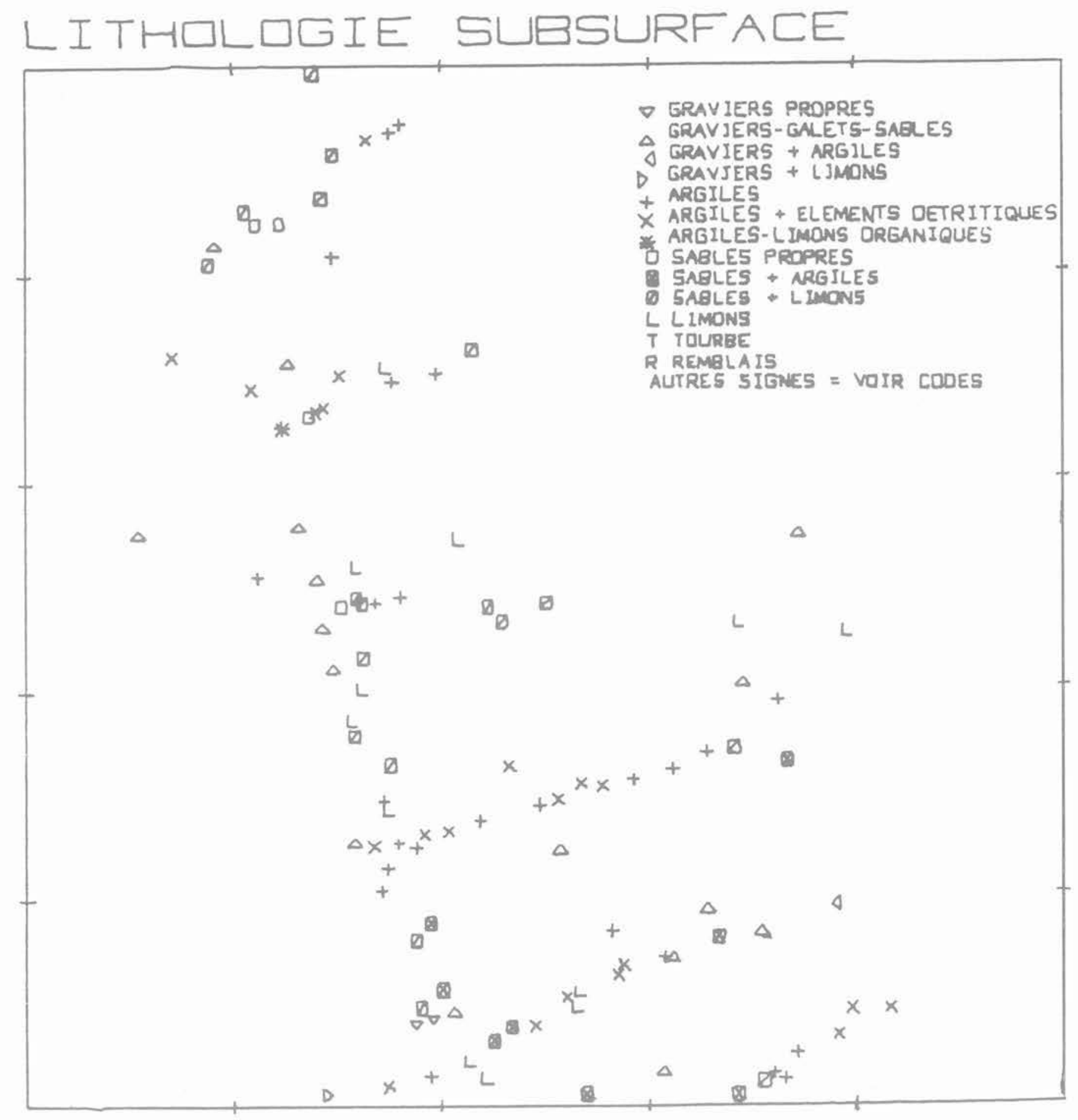

Fig. 4 Exemple d'édition graphique réalisée par le programme GEOL : nature du sous-sol sous la terre végétale aux environs de Voreppe entre les coordonnées Lambert :

$X=856-861(\mathrm{~km})$

$Y=335 \cdot 340(\mathrm{~km})$

\subsection{Résultats du traitement informatique}

La première phase consiste à choisir une zone géographique incluant la partie à étudier et à y rechercher l'existence de sondages. Les résultats de cette opération sont donnés par le programme IMPR 1. Dans un deuxième temps, IMPR 2 donne les coupes des sondages intéressants (voir sur la figure 5 un extrait de la liste des 65 sondages retenus). La nappe est toujours indiquée à plus de $2,50 \mathrm{~m}$ de profondeur dans ce secteur.

La sortie graphique par le programme POZON (fig. 6) montre la distribution irrégulière des sondages du secteur. La zone à urbaniser apparaît clairement en blanc au centre droit de la figure.

Les résultats donnés par ISOPA indiquent que l'épaisseur des sols fins superficiels diminue globalement de l'est vers l'ouest ce qui apparaît bien sur le profil est-ouest établi à l'aide du programme COUPE (fig. 7 a).

Par ailleurs, une autre coupe N.-S. passant par des sondages plus profonds (fig. $7 \mathrm{~b}$ ) permet de dégager l'existence, dans la partie est, de niveaux tourbeux d'épaisseur non négligeable. 


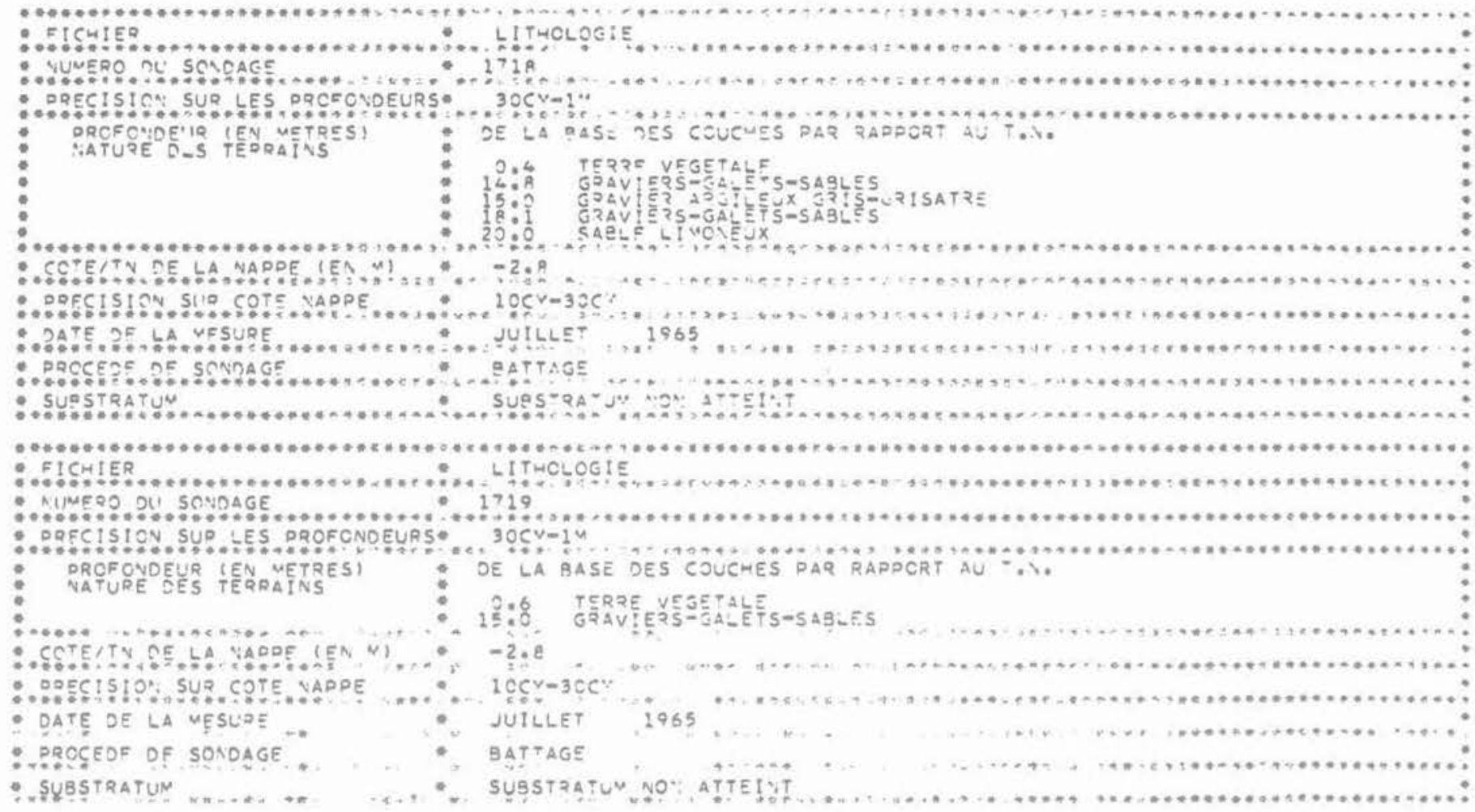

Fig. 5 Exemples de coupes de sondage dans le secteur d'Eybens

Fig. 6 Plan de position des sondages dans le secteur d'Eybens (utilisation du programme POZON)

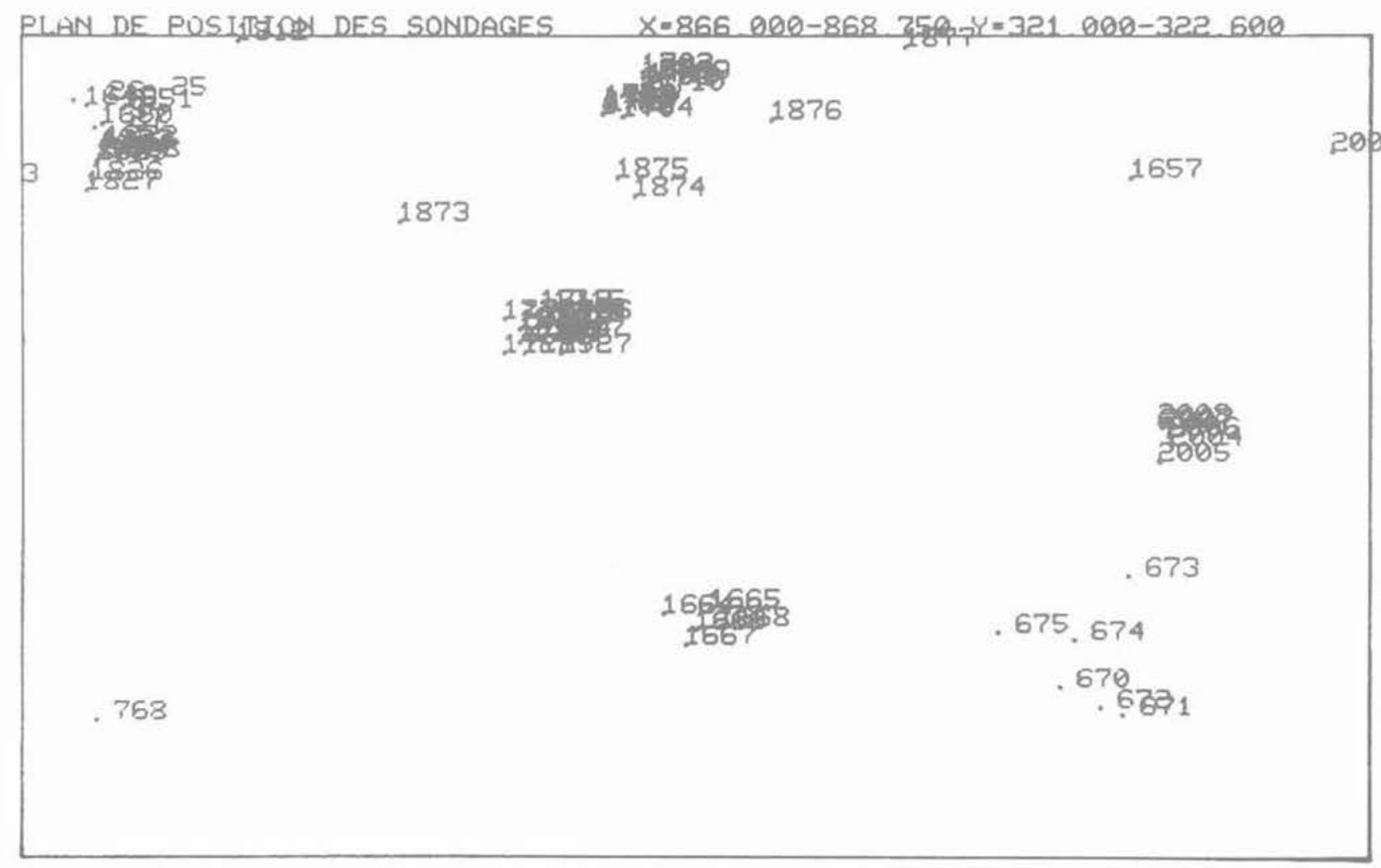




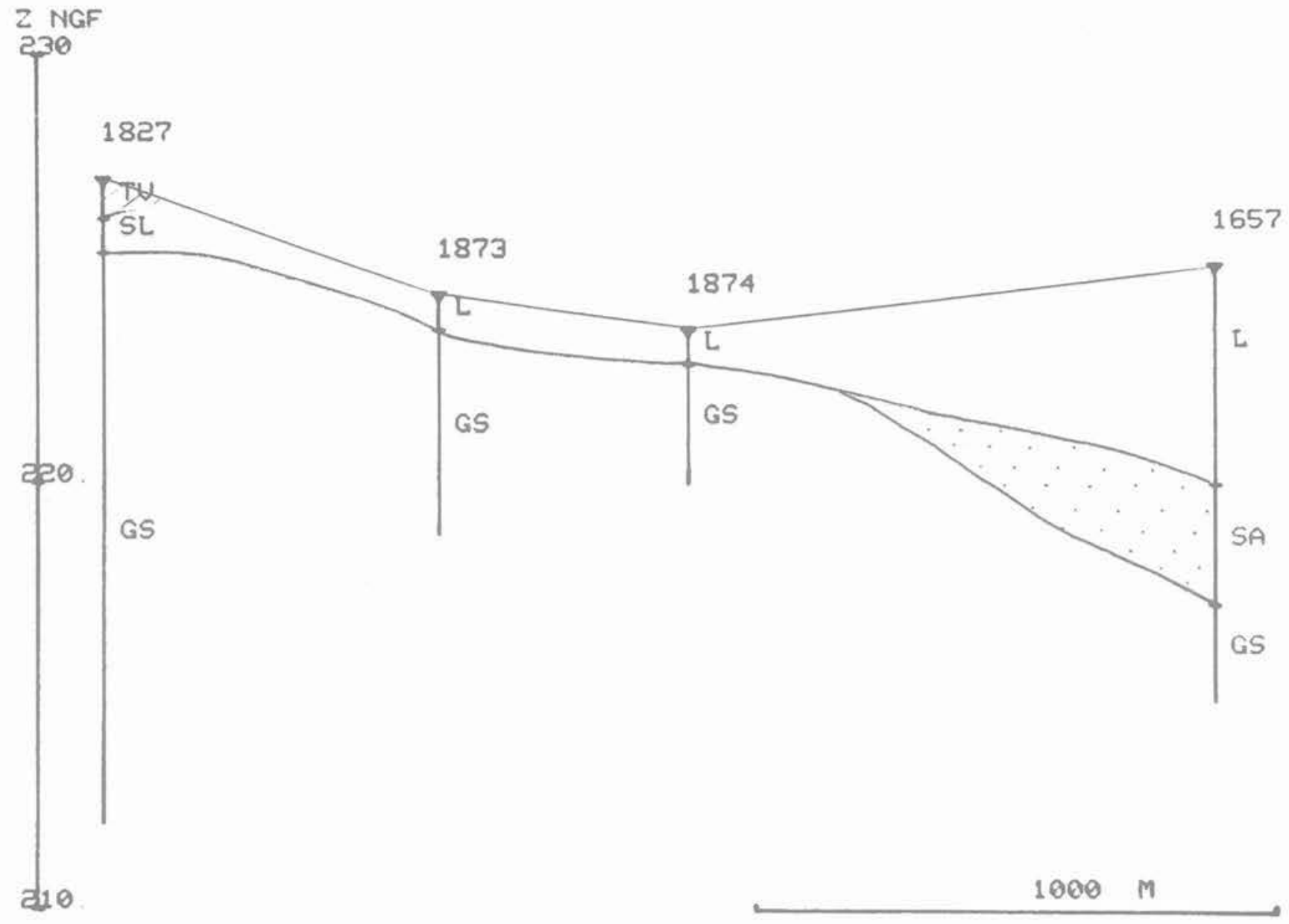

Fig. $7 a$ Coupe E-W interprétée

Fig. 7 Utilisation du programme COUPE pour l'exemple d'Eybens. L'extrapolation entre sondages est laissée à l'appréciation du géotechnicien

\subsection{Synthèse des informations}

L'analyse des données précédentes, que l'outil informatique a permis d'obtenir rapidement, permet une interprétation globale de la structure du site et aide à la définition d'un programme de reconnaissance adapté.

La diminution de l'importance des sols fins de surface de l'est vers l'ouest coilncide avec la présence à l'ouest de la zone d'un petit cône de déjection. L'existence de niveaux tourbeux dans le remplissage alluvial de l'Isère est par ailleurs bien connue. Les sondages étudiés laissent supposer des niveaux assez discontinus.

Après cette recherche préliminaire il apparaît donc que les reconnaissances complémentaires devront porter sur :

- l'épaisseur des sols fins superficiels et la profondeur de la nappe qui conditionnent la nature des fondations à prévoir et peut permettre d'établir un zonage urbanistique de la zone (phase 1);

- la caractérisation des niveaux tourbeux profonds qui pourraient entrainer des tassements différentiels au droit des constructions importantes (phase 2).

\section{Conclusions}

La banque de données réalisée est un outil précieux permettant d'accéder rapidement à des informations géotechniques préalablement analysées, critiquées et codifiées. Les programmes d'édition graphique facilitent la réalisation de cartes et de coupes concernant un secteur particulier du remplissage alluvial étudié. Les documents édités sont toujours contrôlés et interprétés par un intermédiaire géotechnicien ayant une connaissance réelle du contexte géologiques régional. Les informations produites sont particulièrement utiles pour dégager les grandes lignes de la structure du sous-sol et définir le programme de reconnaissance spécifique pour tout projet d'aménagement nouveau.

Sur le plan de la gestion des données, se pose le problème de l'alimentation de la banque en informations nouvelles. Cette actualisation suppose en - particulier la définition d'une convention avec les organismes qui sont à la fois détenteurs d'informations nouvelles et utilisateurs potentiels de la banque (bureaux d'études géotechniques, grandes administrations, etc.). 
16641667

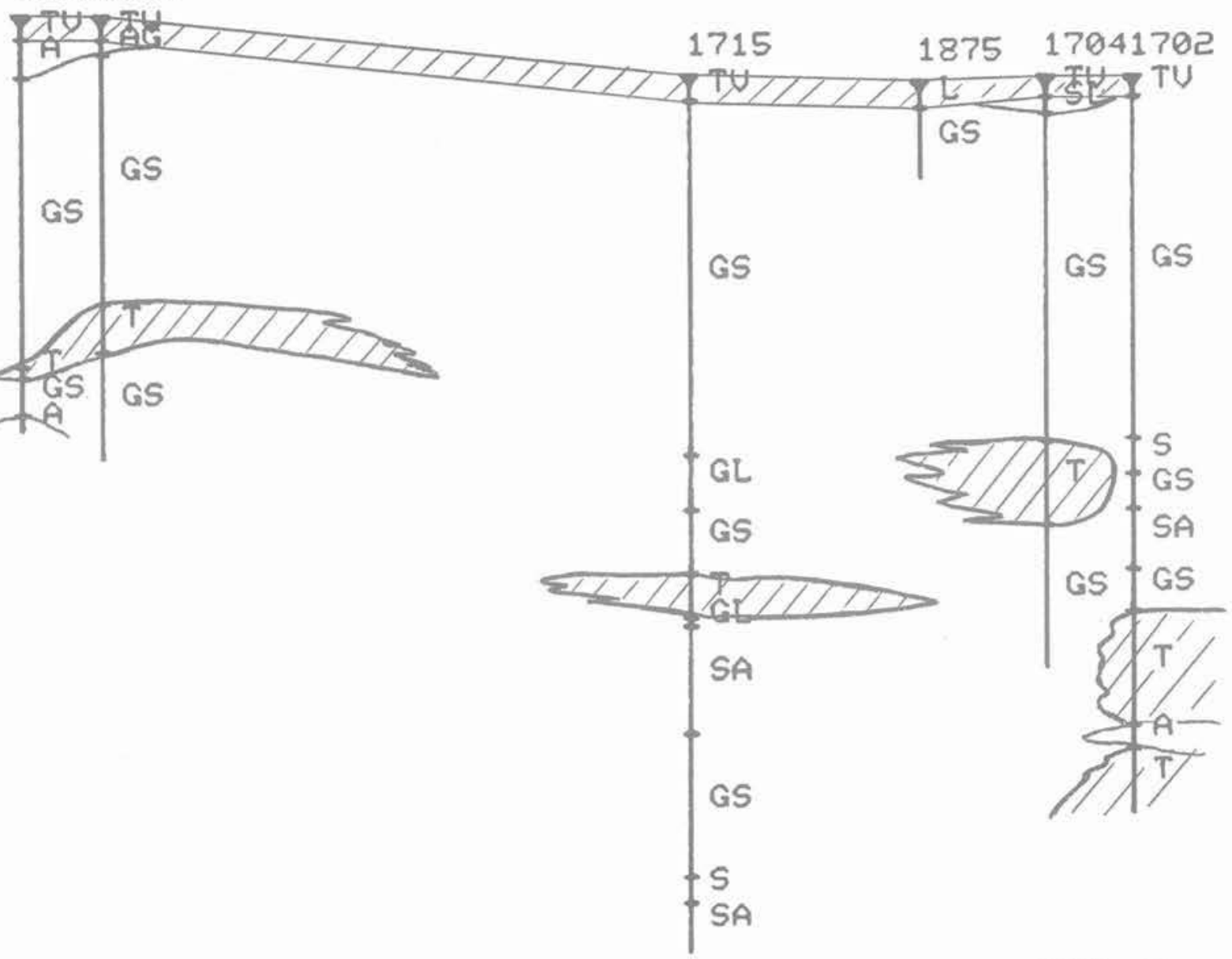

Fig. $7 b$ Coupe N-S interprétée

\section{Références bibliographiques}

Biguenet, G., Dayre M. et Favre J. L. (1970), Essai de cartographie géotechnique de Grenoble et de ses environs. C.R. $1^{\text {er }}$ Congrès int. de I'A.I.G.I. Paris, p. $930-941$.

Bozonnet J. P. (1978). Géologie de I'Aménagement. Étude géologique et géotechnique du moyen Grésivaudan (Isère). Thèse doct. $3^{\circ}$ cycle, Univ. de Grenoble, 158 p. et 16 cartes.

Bozonnet J. P., Antoine P. et Letourneur J. (1978). Cartographie géotechnique de la région grenobloise, $6^{\circ}$ R.A.S.T., Orsay, p. 66.

Couturier B. (1974). Contribution à l'étude géologique, hydrogéologique et géotechnique du bas Grésivaudan (Isère). Thèse $3^{\circ}$ cycle, Univ. de Grenoble, $173 \mathrm{p}$.

Fourneaux J. C. (1976). Les formations quaternaires de la vallée de I'Isère dans I'ombilic de Grenoble. Géol. Alpine, t. 52, p. 31-72.
Margalhan-Ferrat H. (1975). Contribution à l'étude géologique, hydrogéologique et géotechnique de la cluse de I'Isère. Thèse $3^{\circ}$ cycle, Univ. de Grenoble, $128 \mathrm{p}$.

Mercieca G. (1977). Constitution d'un fichier géologique et géotechnique sur ordinateur. Application à l'étude du remblaiement alluvial de la vallée de l'Isère. Thèse $3^{\circ}$ cycle, Univ. de Grenoble, $153 \mathrm{p}$.

Mercieca G., Biguenet G., Antoine P. et Talloni S, (1978). Essai de traitement informatique appliqué à la cartographie géotechnique d'un milieu alluvial (environs de Grenoble - Alpes Françaises). $3^{\circ}$ Congrès int. A.I.G.I., Madrid, Sessions spéciales, p. 176-183.

Talloni S. (1978). Contribution à l'étude géotechnique du sillon subalpin entre Grenoble et Vif. Thèse $3^{\circ}$ cycle, Univ. de Grenoble, 317 p.

Tachker Y. (1973). Procédures administratives pour la délimitation des zones de risques naturels dans le département de l'Isère. Symp. nat. « Sol et sous-sol et sécurité des constructions ", Cannes, 1, p. 377-383. 
